\title{
Tratamiento del paciente cannábico
}

\author{
Solé Puig, J. \\ Psiquiatra, Cruz Roja, Universidad de Barcelona. \\ Correspondencia: Dr. Josep Solé. Creu Roja Toxicomanies. c/ Junta de Comerç 10. 08034 Barcelona \\ tel.9331812188956jsp@comb.es
}

\section{Resumen}

Se ofrece el tratamiento sintomático de las consecuencias psiquiátricas del consumo de cannabis, el tratamiento de cesación del hábito, y la significación de los controles de orina. Sobre el tratamiento sintomático se compara la semivida de eliminación de los cannabinoides y la de algunos antipsicóticos. Por vez primera, al menos en español, se sistematiza la terapia de cesación del hábito cannábico, teniendo como referencia su homóloga del hábito tabáquico. Se alude al contexto de uso recreativo, a la importancia del ambiente de consumo, y a las interacciones farmacológicas deseadas por los policonsumidores. Se define la cesación del hábito cannábico como terapia psicológica ambulatoria basada en el counselling. Comprende los abordajes individual, grupal y familiar. En este último se distingue entre intervención informativa e intervención terapéutica. Por último, se considera la significación de los controles de orina en el proceso terapéutico. Las conclusiones son: que además de ofrecer tratamiento psiquiátrico sintomático hay que ofrecer tratamiento de cesación del hábito. Que este último requiere competencias profesionales de tipo counselling e incluso psicoterapia, y que los controles de orina son válidos como complemento con fines terapéuticos, pero nunca fiscalizadores por la lenta eliminación de los cannabinoides.

Palabras clave: cannabis, tratamiento, interrupción, controles de orina.

\section{INTRODUCCIÓN}

A I alba del tercer milenio, el debate sobre las drogas parece centrarse en dos productos que se fuman: el taba-

\section{Summary}

The symptomatic psychiatric treatment of cannabis abuse, the cessation treatment of the habit, and the significance of urinalysis are described. The respective elimination half-lifes of both cannabinoids and some antipsychotics are compared. At least in Spanish, this is the first time when the cessation treatment of the cannabis habit is proposed and systematised. The reference is clearly its homologous in tobaccoism. Context factors as recreative use, relevance of the setting in which cannabis use takes place, and the desired drug interactions of polyusers are considered. Cannabis habit cessation treatment is defined as psychological outpatient therapy technically based on counselling. Individual, group and family approaches are described. The latter includes information and/or therapy interventions. The significance of urinalysis in the treatment process is considered. Conclusions are: besides psychiatric treatment of the symptoms caused by cannabis abuse, cessation treatment of the cannabis habit must be offered. The latter requires as well specific professional competence, to be defined as counselling or even psychotherapy. Urinalysis controls are useful as a complementary treatment instrument, but given the slow elimination of cannabionids they are not indicated for surveillance purposes.

Key words: cannabis, treatment, cessation, urinalysis control.

co y el cannabis (Solé Puig, 2000a). La prohibición estadounidense está acabando allí con el hábito nicotínico y se lo está poniendo cada vez más difícil al fumador europeo. También en España, y pese a la alta prevalencia 
de tabaquismo, la sensibilidad antitabaco va abriéndose camino. En contraste, el cannabis es objeto de una campaña permanente de promoción social en ambas riberas del Atlántico. Desde la óptica de la droga, los cigarrillos de tabaco siguen siendo, con diferencia, la sustancia de abuso más consumida del mundo, y los cigarrillos o porros de marihuana o de hachís siguen representando lo mismo en el campo ilegal. En términos de mercado, los primeros parece que irán a menos en el futuro, y los segundos quizá sean más tolerados socialmente, por lo menos en Europa. Estamos, pues, ante dos consumos que se fuman, que son masivos, pandémicos, y cuyo status social-legal se diría que está en trance de aproximarse para quizá acabar convergiendo.

Cannabis y tabaco se hallan, por tanto, en un campo de fuerzas marcado por el antagonismo existente -lo cual es típico en drogasentre la legalidad o la tolerancia y la prohibición. La prohibición como método de prevención por contención legal frente a los psicotropos potencialmente peligrosos tiene su paradigma, concretado en el alcohol, en la prohibición estadounidense entre los años 1919 y 1934. Quince años de prohibición del alcohol supusieron menor tasa de fallecimientos por cirrosis hepática y mayor alarma social ante el gangsterismo. Tres años después, en 1937, los americanos pusieron en vigor la Marijuana Tax Act, que transfería la prohibición al cannabis. Desde entonces, los países occidentales asumieron esta prohibición, aunque con matices. Así, en los propios Estados Unidos hay once estados, California y Nueva York entre ellos, en donde desde 1976 el consumo se halla despenalizado. Europa, desde la posguerra, hizo suya la prohibición americana. En 1992, el Parlamento Europeo se pronunció contra la legalización de psicotropos distintos del tabaco, el alcohol y los psicofármacos adictivos (barbitúricos, benzodiacepinas). De los Países Bajos se denunció su carácter de oasis de la droga, y se les instó a alinearse con en la política general prohibicionista. En Holanda y desde 1978 está permitida la posesión de hasta treinta gramos de cannabis para uso personal. Hay allí unas dos mil coffe-shops donde se vende y se consume marihuana. En España, fumar porros goza de una tolerancia social indiscutible. Es notoria, también, la militancia antiprohibicionista del lingüista Noam Chomsky y del Premio Nobel Milton Friedman, fundador de la Drug Policy Foundation y para el cual las únicas leyes a las que debieran someterse todo tipo de drogas son las del mercado.

Hay, por tanto, sobrados ejemplos en Europa y Estados Unidos de la masiva coexistencia de fumar tabaco y fumar cannabis. Desde esta perspectiva sorprende que dispongamos de un amplio caudal de información técnica para ayudar al nicotindependiente a que deje de fumar y que no suceda lo mismo para el caso del hábito cannábico. Más allá del debate sobre si consumir cannabis genera dependencia física o no, hay consumidores de porros necesitados de ayuda para abandonar su hábito; es evidente que este tipo de ayuda técnica sólo está disponible para quienes desean abandonar el tabaquismo. En este trabajo, que pretende ser consecuentemente holista, revisamos el tratamiento sintomático que suelen recibir las secuelas psiquiátricas por abuso de cannabis y nos centramos en la propuesta de tratamiento de cesación del hábito cannábico. Esto último es novedoso y la idea es que debería ser ofertado en los ambientes asistenciales especializados en drogodependencias. Finalmente, intentamos situar el papel que tienen los controles de detección de cannabinoides en orina a lo largo del proceso terapéutico y desaconsejamos dichos análisis de orina cuando se hacen con fines fiscalizadores, y ello en razón de la lenta cinética de los cannabinoides. La discusión incide en la naturaleza ambivalente del cannabis y de todas las sustancias de abuso, un conocimiento que es básico y que todo terapeuta debería tener siempre presente durante el proceso de ayuda al consumidor. Las conclusiones recapitulan las aportaciones del trabajo. 


\section{TRATAMIENTO SINTOMÁTICO}

En los países de nuestro entorno se considera que el consumo de cannabis, por si mismo, no requiere ningún tratamiento específico. La razón estriba en que el síndrome de abstinencia, si existe, no interfiere significativamente la vida cotidiana del usuario. La dependencia física, si existe, no da problemas. Por ello no suele haber necesidad de prescribir medicamentos de tolerancia cruzada con el THC. Incluso se considera desaconsejable si deseamos que el paciente aprenda a afrontar las consecuencias de sus actos sin tener que refugiarse en soluciones farmacológicas.

En cambio, sí que debe conocerse el tratamiento de los cuadros psicopatológicos por consumo cannábico, para cuya evaluación nos valdremos de los libros de referencia diagnósticos DSM-IV (cuadro 1 y 2) o la CIE10 (cuadro 3 y 4).

En caso de psicosis deben prescribirse neurolépticos. De primera intención quizá optemos por los de última generación como la risperidona, la olanzapina o el flupentixol, por el plus de tolerabilidad de que puedan gozar. Sin embargo, también recomendamos la clorpormacina en dosis alrededor de 75 $\mathrm{mg} / \mathrm{dia}$, pues presenta una semivida de eliminación (t1/2) lenta, unas 30 horas, siendo todavía el patrón de referencia de los antipsicóticos. Se obtienen también excelentes resultados con tioridazina (t1/2 también de unas 30 horas) y levomepromazina (t1/2 entre 16 y 77 horas). Ello es extensible también al

Cuadro 1. Criterios diagnósticos del DSM-IV para F12.00: Intoxicación cannábica

\section{A) CONSUMO RECIENTE DE CANNABIS}

B) IMPORTANTES CAMBIOS DESADAPTIVOS CONDUCTUALES O PSICOLÓGICOS (por ej. coordinación motora alterada, euforia, ansiedad, sensación de tiempo enlentecido, juicio alterado, retraimiento social) DESARROLLADOS DURANTE EL, OTRAS POCOTIEMPO DE, CONSUMO DE CANNABIS.

C) DOS (O MÁS) DE LOS SIGNOS SIGUIENTES, desarrollados dentro de las2 horas posteriores al uso de cannabis:

1. INYECCIÓN CONJUNTIVAL

2. APETITO AUMENTADO

3. BOCA SECA

4. TAQUICARDIA

D) LOS SÍNTOMAS NO SE DEBEN A UNA CONDICIÓN MÉDICA GENERAL NI PUEDEN EXPLICARSE MEJOR MEDIANTE OTROTRANSTORNO MENTAL.

*especificar si: F12.04 con alteraciones perceptivas

Cuadro 2. Criterios diagnósticos del DSM-IV. Trastornos relacionados con cannabis

TRASTORNOS POR CONSUMO DE CANNABIS

F12.2X Dependencia de cannabis

F12.1 Abuso de cannabis

TRASTORNOS POR CONSUMO DE CANNABIS

F12.00 Intoxicación por cannabis

F12.04 intoxicación por cannabis, con alteraciones perceptivas

F12.03 delirium por intoxicación con cannabis

F12.XX Trastorno psicótico inducido por cannabis

.51 con ideas delirantes, de inocio durante la intoxicación

.52 con alucinaciones, de inicio durante la intoxicación

F12.8 trastorno de ansiedad inducido por cannabis

F12.9 trastorno relacionado con cannabis no especificado 


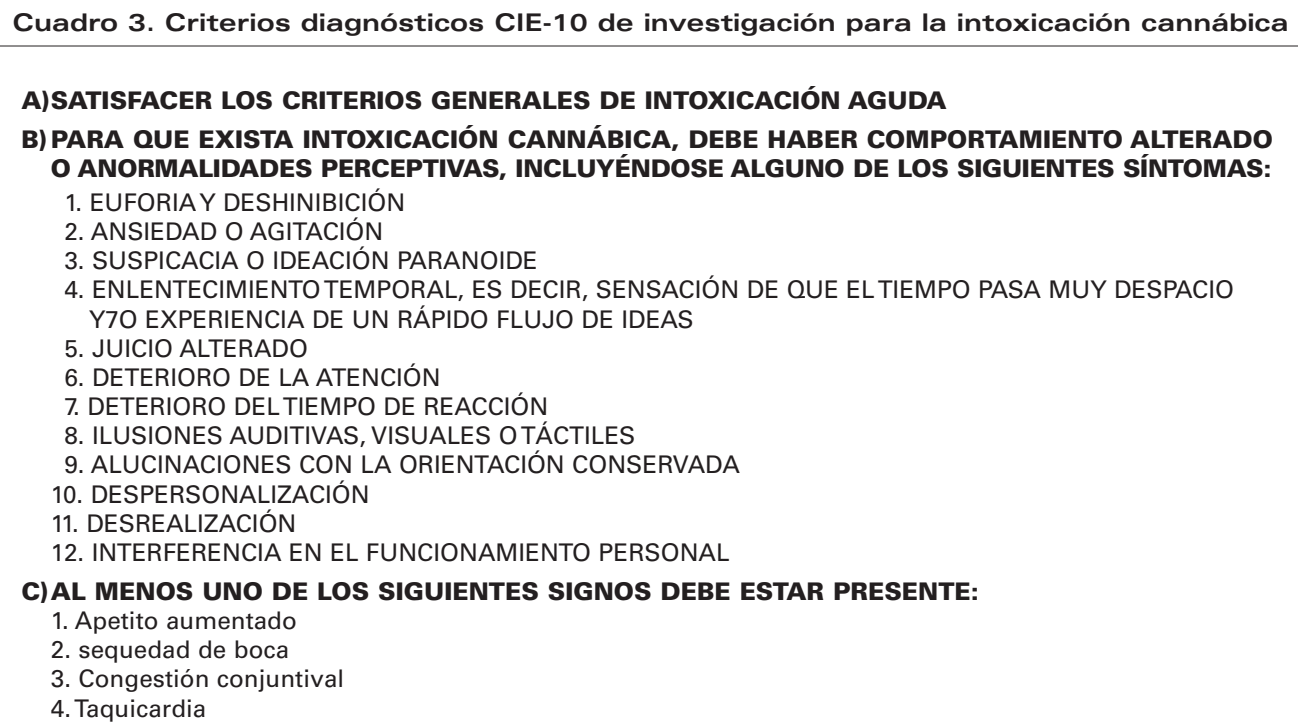

C)AL MENOS UNO DE LOS SIGUIENTES SIGNOS DEBE ESTAR PRESENTE:

1. Apetito aumentado

2. sequedad de boca

3. Congestión conjuntival

4. Taquicardia

\begin{tabular}{|c|}
\hline Cuadro 4. CIE-10 versus DSM-IV \\
\hline F.12.03 INTOXICACIÓN CANNÁBICA CON DELIRIUM \\
\hline $\begin{array}{l}\text { EI DSM-IV INCLUYE EL DELIRIUM POR INTOXICACIÓN CANNÁBICA CON CRITERIOS GENÉRICOS PARA } \\
\text { TODAS LAS SUSTANCIAS }\end{array}$ \\
\hline F.12.3 SÍNDROME DE ABSTINENCIA (SÓLO CIE-10) \\
\hline EL DSM-IV INCLUYE EL SÍNDROME DE ABSTINENCIA CANNÁBICO \\
\hline
\end{tabular}

haloperidol, con una t1/2 algo menor, de unas 18 horas. La t1/2 de la 9-hidroxi-risperidona, el metabolito activo de la risperidona, es de unas 24 horas, suficiente también para neu- tralizar el efecto psicotomimético de los cannabinoides. En la tabla 1 comparamos la semivida de los cannabinoides y la de tres neurolépticos.

\begin{tabular}{|c|c|c|c|}
\hline Cannabinoides & Clorpromazina & Tioridacina & Haloperidol \\
\hline 14 a 40 & $30(+/-7)$ & 26-35 & $18(+/-6)$ \\
\hline
\end{tabular}

Dada la lenta semivida de eliminación de los cannabinoides parece preferible decantarse por neurolépticos de farmacocinética similar, que además son los más utilizados. No obstante, la psicotomímesis cannábica se manifiesta durante las primeras horas siguientes a la inhalación o ingesta. Luego la persistencia prolongada de cannabinoides en sangre deja de tener significación comportamental o subjetiva. De ahí que también neu- rolépticos de vida media más breve, como la perfenacina, loxapina, y clozapina, con t1/2 que no rebasan las 12 horas, se adapten bien al periodo a cubrir y den una buena respuesta antipsicótica. En la práctica todos los neurolépticos pueden ser indicados. Lo importante es que el médico prescriba el neuroléptico con el que se halle más familiarizado. En el momento de salir a la luz esta monografía son de primera elección los antipsicóticos 
olanzapina, risperidona y flupentixol, por un perfil de toleranacia acorde con los estándares actuales.

El diagnóstico de delírium cannábico, raro o quizá inexistente, aconsejaría la monitorización hidroelectrolítica del enfermo, si bien el riesgo en este sentido sería claramente menor que en caso de un delírium tremens. El tratamiento de urgencia puede ser la administración intramuscular de 25-50 mg de clorpromacina o de 5-10 mg de haloperidol, aunque también de 10 mg de diacepán o 50 de clorazepato si se juzga que la pronta y simple ansiolisis ha de bastar.

Puede estar indicado administrar también antipsicóticos en el discutido síndrome de abstinencia por cannabinoides, pues hay veces que aparecen síntomas de la serie psicótica y entonces vale la pena prevenir complicaciones en este sentido. De primera intención no suele emplearse antidepresivos, aunque se ha descrito el uso de tricíclicos como la desipramina (Tennant, 1986) tricíclico inhibidor de la recaptación de noradrenalina. La desipramina no se halla comercializada en España, pero al ser un metabolito de la imipramina, puede utilizarse ésta a todos los efectos. De todos modos, actualmente es recomendable prescribir de primera intención inhibidores selectivos de la recaptación de serotonina como fluoxetina, paroxetina, fluvoxamina, sertralina y citalopram, por su mayor tolerancia. Aunque apenas se dispone de experiencia con venlafaxina, nefazodona y mirtazapina, su uso en medicina de la adicción también parece prometedor.

En cuanto a las reacciones adversas, ansiosas, por intoxicación cannábica aguda, o sea los "malos viajes" (bad trips), sigue siendo válido el consejo de Smith (1968) en su trabajo ya clásico, cuando proponía administrar una pequeña dosis de tranquilizante via oral y simultáneamente seguir el método de la charla (talk down) relajada con el paciente, sin otro propósito que calmarle la angustia. Sólo en algún caso habrá de recurrirse a la administración de benzodiacepinas.

\section{CESACIÓN DEL HÁBITO CANNÁBICO}

La adicción a la marihuana y al hachís conlleva alteraciones en las esferas cognitiva, emocional (estado de ánimo) y psicosocial (Solowij, 1998) beneficiarias de diagnóstico y tratamiento y con una evolución clínica predecible. También con el cannabis, y tal como siempre ocurre en caso de problemas por consumo de sustancias, la conceptualización de enfermedad puede conducir a malpraxis si no se abordan las condiciones psicosociales, interpersonales y de rol, que contextualizan el consumo, abuso, o dependencia de dicha sustancia.

El cannabis se asocia comúnmente al uso recreativo (cuadro 5 ). Se ha demostrado que el ambiente (setting) en que el consumo tiene lugar va a determinar en gran manera sus posibles efectos. Existe escasa literatura acerca de los efectos del cannabis en la conducta social, lo que se explicaría por las dificultades metodológicas que comporta dicho análisis. Para ello sería necesario recrear in vitro el entorno en que se desarrolla el uso, con sujetos voluntarios que consumieran cannabis y en dosis estrictamente controladas. En la práctica, dichos estudios se han basado en cuestionarios o entrevistas semiestructuradas, con sujetos que en el momento de la evaluación estaban, o parecían estar, abstinentes. No había, por tanto, un entorno similar al del consumo y tampoco había un grupo control. Dichas deficiencias técnicas hacen que sea muy difícil la extrapolación de los resultados obtenidos.

Cuando se han creado las condiciones adecuadas controlándose las distintas variables que intervienen, se ha observado que los efectos farmacológicos de las preparaciones cannábicas dependen en gran medida de variaciones situacionales, que los efectos de dosis relevantes de la droga no siguen un patrón lineal ,y que una misma cantidad de la sustancia puede presentar efectos muy diferentes, en función de si se toma en un lugar familiar o extraño, en compañía de amigos o desconocidos, y si se asocia o no a consumos de alcohol y demás psicotropos (cuadro 6 ). 


\begin{tabular}{|l|}
\hline \multicolumn{1}{|c|}{ Cuadro 5. Terapia de cesación del hábito cannábico } \\
\hline \multicolumn{1}{|c|}{ Cannabismo } \\
\hline 1) CONCEPTUALIZACIÓN COMO ENFERMEDAD \\
2) USO RECREATIVO \\
Escasos estudios sobre los efectos del cannabis en la conducta social \\
- Importancia SETTING de consumo \\
* Dospectativa del sujeto \\
\hline
\end{tabular}

\begin{tabular}{|l|}
\hline \multicolumn{1}{|c|}{ Cuadro 6. Contexto del consumo cannábico } \\
\hline \multicolumn{1}{|c|}{ Status de prohibición/minimización } \\
\hline INTERACCIONES FARMACOLÓGICAS DESEADAS \\
2) USO RECREATIVO \\
- PARA NEUTRALIZAR EFECTOS: cannabinoides, heroína, alcohol o benzodiacepinas tras consumo de coca- \\
ína O anfetaminas. \\
- PARA PROLONGAR LOS EFECTOS: cannabinoides después de heroína. \\
PARA POTENCIAR LA SENSACIÓN DE EMBRIAGUEZ: cannabinoides y alcohol. \\
\hline
\end{tabular}

Al margen de estas consideraciones previas, que nos ayudan a resituar el tema desde una perspectiva más amplia, aquí nos centraremos en los aspectos terapéuticos de este tipo de consumo. En primer lugar, cabe decir que una cosa es tratar las secuelas próximas o remotas del cannabismo, y otra es tratar el hábito de fumar porros de hachís o marihuana. Esto último es precisamente la terapia de cesación anticannábica, que presenta una notable dificultad psicológica, perfectamente parangonable a la propia de la terapia antitabáquica. En este sentido, son de sobras conocidos los obstáculos motivacionales que un dependiente de nicotina debe salvar, tanto si decide cesar de fumar con asistencia técnica o por si solo.

Las estadísticas acerca de los hábitos de consumo de la población general nos muestran que, en su historia natural, el consumo de cannabis decrece con la edad (cuadro 7). Esto parece cumplirse menos en los casos en que el consumo cannábico haya supuesto el primer peldaño en la toma de contacto con otras sustancias ilegales. En efecto, nos estamos refiriendo al clásico, aunque siempre polémico, concepto de escalada en el consumo de drogas, en la que de forma clara interviene una presión grupal tendente a menospreciar el riesgo de posibles dependencias posteriores.

Además de estos dos factores reforzantes del consumo de cannabis, es decir, la sustancia en si misma (refuerzo primario) y el clima grupal inductor (refuerzo secundario), en el caso del hábito cannábico hay que añadir un obstáculo más, quizá el principal: que quien fuma cigarrillos cannábicos o porros no suele sentir la necesidad de dejar de hacerlo. Aun en el caso de que el fumador de cannabis

\begin{tabular}{l} 
Cuadro 7. Tratamiento del cannabismo (1) \\
\hline - Secuelas del cannabis. \\
TÉbito de fumar cannabis: \\
A)CONSIDERACIONES PREVIAS: \\
- El consumo decrece con la edad \\
\hline
\end{tabular}


aqueje molestias y refiera síntomas, normalmente no lo relacionará con su consumo. Ello sucede porque, en un contexto de uso múltiple de psicotropos, los cannabinoides casi nunca se incluyen entre las principales drogas nocivas. Pero el consumo cannábico puede conllevar adversidades. La negación de las mismas por parte de quien las sufre es simplemente un ejemplo más de la distorsión atributiva que típicamente muestra cualquier consumidor de sustancias de abuso: nicotina, alcohol, heroína, cocaína, etc. (cuadro 8).

\begin{tabular}{|l|} 
Cluadro 8. Tratamiento del cannabismo (2) \\
\hline B) DIFICULTADES \\
- REFUERZO PRIMARIO (SUSTANCIA) \\
- REFUERZO SECUNDARIO (SETTING GRUPAL INDUCTOR) \\
CBSTÁCULOS MOTIVACIONALES: DISTORSIÓN ATRIBUTIVA \\
C) CARACTERÍSTICAS DE LA INTERVENCIÓN \\
1. TERAPIA PSICOLÓGICA EN RÉGIMEN AMBULATORIO \\
2. POBLACIÓN DIANA: ADOLESCENTESY ADULTOS JÓVENES PREVENCIÓN SECUNDARIA \\
3. ESTILOTERAPÉUTICO NO DIRECTIVO: MÉTODOS INDIRECTOS, ACTITUD DE TOLERANTE ESPERA \\
\hline
\end{tabular}

¿Es entonces posible la terapia de cesación de fumar cannabinoides? Sí, a condición de dotar de suficiente contenido psicopedagógico a la intervención terapéutica, considerando a priori que la mayoría de los consumidores pueden abandonar el hábito sin someterse a ningún tipo de tratamiento. Sólo aquéllos que han decidido abandonar la sustancia y no lo han conseguido por sus propios medios serán los que realizarán algún tipo de demanda, normalmente en centros públicos especializados o bien en gabinetes privados.

En general, si la implicación con la droga no es muy profunda y se cuenta con un mínimo de recursos personales, lo más aconsejable es optar, de entrada, por modalidades poco intrusivas como el tratamiento ambulatorio o bien la participación en grupos de autoayuda para cesación de cannabismo, si éstos existen. Sólo en caso de consumos paralelos de otro tipo de drogas, o bien si aparece sintomatología psiquiátrica, habría de plantearse otro tipo de intervención. La opción preferible en estos supestos sería la de la asistencia en régimen cerrado, esto es, un recurso de tipo residencial que dotara de mayores garantías al proceso de deshabituación, por lo menos en sus inicios (USDHHS, 1998).

Existen diferentes abordajes terapeúticos para tratar este tipo de drogodependencia, pero cualquier tipo de intervención orientada al cambio debería partir de las siguiente premisas:

1) El abandono del hábito cannábico provendrá de una terapia principalmente psicosocial, en régimen ambulatorio, similar a la terapia de cesación del hábito tabáquico.

2) Se centrará en pacientes adolescentes y adultos jóvenes, los principales afectados, como forma de prevención secundaria. Aunque es evidente que tendrá en cuenta cualquier franja de edad.

3) Los terapeutas deberán evitar dar pie a que los pacientes adolescentes les identifiquen automáticamente con los padres. Si éstos desean intervenir o necesitan ayuda, es mejor derivarlos a otro equipo terapéutico.

4) Los terapeutas han de ser concretos en los contenidos. La interacción gestual, complementaria de la verbal, es importante. El estilo de interacción terapeuta /paciente no debe ser sólo vertical, pero tampoco falsamente horizontal. Si es factible, cabe echar mano de juego de roles, manualidades, instrumentos musicales, dibujos, collages, etc. Cuanta mejor formación psicoterapéutica tenga el profesional concernido, tanto más beneficiado resultará el paciente (Solé Puig, 1995).

5) El objetivo es, también aquí, la responsabilización del fumador de cannabis en relación a sí mismo y a los demás. Es meta de 
la terapia psicosocial desactivar prejuicios y falsas atribuciones, así como clarificar el campo motivacional de cada paciente para que sus intenciones de salud se conviertan en realizaciones concretas.

6) El aquí y ahora del paciente importa más que su pasado. Siempre es meta de la terapia despertar expectativas de futuro en el paciente, tratando de activar sus propios recursos personales.

La terapia de cesación del hábito cannábico, como la del tabaquismo, requiere un estilo terapéutico no directivo. Al principio, el terapeuta opta por una pasividad aparente, omitiendo hacer preguntas y planes. Quien consume cannabis no suele expresar quejas al respecto o no es consciente de ellas. De ahí que no suela haber demanda de ayuda para dejar de fumar porros. Esta realidad, tan distinta de la del heroinómano que urge vehementemente la asistencia, le impone al terapeuta del hábito cannábico una actitud receptiva y de tolerancia, sin plazos previos ni fases formalizadas.

La terapia de cesación anticannábica que juzgamos posible, en la práctica únicamente indicada si hay fuerte consumo diario durante años y que de hecho sólo implementamos en las escasas ocasiones que se solicita, puede esquematizarse como sigue.

El programa comienza con la desintoxicación ambulatoria del paciente, cosa que significa abstinencia de preparaciones cannábicas y eventual administración de ansiolíticos. Se aprovecha así la tolerancia cruzada entre cannabinoides y benzodiacepinas para fines terapéuticos. Aunque en cesación antitabáquica también pueden prescribirse benzodiacepinas a pequeñas dosis, ahí el efecto beneficioso no descansa en la tolerancia cruzada, sino en la neutralización en sistema nervioso central del efecto estimulante de la nicotina (cuadro 9).

\begin{tabular}{|c|c|}
\hline \multicolumn{2}{|r|}{ Cuadro 9. Tratamiento del cannabismo (3) } \\
\hline \multicolumn{2}{|c|}{ D)FASES DEL PROGRAMA: } \\
\hline \multicolumn{2}{|c|}{$1^{\text {a }}$.DESINTOXICACIÓN AMBULATORIA: } \\
\hline \multicolumn{2}{|c|}{$\begin{array}{l}\text {-ANTICIPACIÓN ACONTECIMIENTOS } \\
\text { - POSIBLE USO DE BENZODIACEPINAS }\end{array}$} \\
\hline \multicolumn{2}{|c|}{$2^{a}$.DESHABITUACIÓN: (1) } \\
\hline \multicolumn{2}{|c|}{ A. TERAPIA INDIVIDUAL: } \\
\hline OBJETIVOS: & $\begin{array}{l}\text { - aumento conciencia de problema } \\
\text { - desactivar prejuicios y falsas creencias } \\
\text { - generar expectativas de futuro }\end{array}$ \\
\hline TÉCNICAS: & $\begin{array}{l}\text { - counseling } \\
\text { - entrenamiento en habilidades sociales } \\
\text { - prevención de recaídas } \\
\text { - refuerzo cts., alternativas: sist. incentivos }\end{array}$ \\
\hline
\end{tabular}

Llegados a este punto hay que recordar que en algunos casos es posible la utilización inicial de técnicas aversivas, para apoyar los primeros momentos de la interrupción del cannabis. Entre las más conocidas mencionaremos la técnica de fumar de forma rápida, también utilizada en el tratamiento del tabaquismo. Entre los inconvenientes de esta técnica se señalan las dificultades de mantenimiento de sus resultados. Ello se debe a que el objetivo inicial es meramente el de la inte- rrupción o disminución de la conducta problema y no la instauración de nuevas formas alternativas de conducta. Por eso, con el fin de lograr buenos resultados tales técnicas aversivas deberían asociarse a otro tipo de intervenciones, de tipo individual o grupal, que desde una vertiente positiva de activación de los propios recursos personales de cada paciente, modificara las atribuciones del sujeto a los efectos de la sustancia. En la práctica, una vez más, rigen los mismos prin- 
cipios que en cesación del tabaquismo, por lo que las técnicas aversivas serán una indicación secundaria o incluso desechada. Si hay demanda del paciente en dicho sentido deberá valorarse su idoneidad en cada caso.

Durante las dos o tres primeras semanas de desintoxicación, el cliente deberá acudir, a diario si es posible, o cuanto más frecuentemente mejor. Es obvio que la ansiolisis farmacológica sólo tiene sentido mientras haya ansiedad. El médico no debe dilatar el periodo de prescripción de benzodiacepinas por el conocido problema de la dependencia, potencialmente grave. Sin embargo, tampoco deberá acortarlo por exceso de alarma ante dicha yatrogenia, pues entonces podría quedar comprometido ya de entrada el éxito del tratamiento.

En cada sesión, si ello es factible, se tomará muestra de orina para detección de THC y otros metabolitos, con el doble propósito de comprobar si efectivamente hay abstinencia y, más que nada, alentar (refuerzo social) al paciente. Como los cannabinoides se metabolizan de forma lenta, el paciente puede comprobar que tras muchos días de haber cesado de fumar porros su organismo todavía es portador de metabolitos cannabinoides. En realidad el THC carboxílico será detectable hasta transcurrido tres semanas e incluso más de un mes. Este hecho suele impresionar desagradablemente al fumador, animándole a que prosiga en la dirección de mantener estable la abstinencia

El terapeuta, además, puede anticipar acontecimientos al paciente, diciéndole por ejemplo que tras la interrupción del hábito cannábico es normal sufrir insomnio. Es un tipo de información que conviene dar para que el paciente reciba un mensaje de dominio técnico que le tranquilice, y que se afiance así su sentido de seguridad en una abstinencia sólo muy recientemente adquirida. Más tarde, llegado el momento en que se comprueba que la orina está limpia (drug free) de cannabinoides, incluso hay muestras de alborozo en el grupo terapéutico, por la expectación generada ante la lenta farmaco- cinética del THC y demás metabolitos cannabionides.

Superada esta primera etapa de la desintoxicación, la monitorización debe prolongarse todo el tiempo necesario. Como referencia sugerimos un año, tiempo durante el cual se aplicarán técnicas de carácter psicológico, de cara a poder favorecer el proceso de deshabituación.

\subsection{Abordaje individual}

Es probablemente la parte más importante dentro del abordaje destinado a la deshabituación, debiéndose considerar los aspectos generales anteriormente comentados. Dentro de las diferentes escuelas psicológicas existentes, nos centraremos en las cognitivoconductuales, las interpersonales, y las psicodinámicas. Según sea la formación del psiquiatra o del psicólogo clínico que hace la terapia, las técnicas de dichas escuelas se aplicarán de forma separada o integrada. La tendencia actual es a integrar las psicoterapias. La psicoterapia se orientará hacia el aumento de la conciencia de problema, normalmente baja, así como a incrementar la motivación, usualmente también escasa, por abandonar definitivamente el consumo. Los tratamientos eficaces incluyen aconsejamiento y orientación, enseñanza de habilidades adecuadas, y refuerzo de la conducta alternativa apropiada (USDHHS, 1997).

El psicoterapeuta puede organizar un sistema de incentivos: pagar deudas, que los padres asignen algún dinero en pago de prestaciones, etc. De esta manera se intenta neutralizar la inercia de pasividad que todavía muestra el paciente, llevándole a avanzar en el programa de rehabilitación. En esta planificación de incentivos ocuparán un lugar clave los allegados. Si éstos no existen, es el equipo terapéutico quien se erige en familia sucedánea, y los mensajes de aprobación/desaprobación logran tener alguna fuerza moduladora en la manera de conducirse el paciente. 
Dentro de este marco psicoterapéutico no puede omitirse hablar del llamado síndrome amotivacional que clásicamente se ha asociado a los consumidores crónicos de cannabis pero que probablemente es inespecífico. Sus características pueden resumirse en las siguientes: pasividad, disminución de las motivaciones cotidianas, y desinterés general. A pesar de que la práctica clínica corroboraría su existencia, hay que señalar que la relación de causa-efecto entre dicho consumo y estos patrones de conducta está lejos de haberse clarificado. Hay que tener presente que algo tan vago como la sintomatología amotivacional podría estar mediatizado por múltiples variables intermedias. Además, la propia definición DSM-IV o CIE-10 de dependencia se fundamenta en la priorización del consumo por encima de todo lo demás. Esto equivale a decir que el drogodependiente está casi exclusivamente motivado por perpetuar su hábito y, por tanto, desmotivado/ amotivado para el resto de los intereses cotidianos. En esta línea, el síndrome amotivacional no sería otra cosa que un sinónimo de dependencia cannábica.

Es más, los cambios generales de comportamiento o actitudinales podrían deberse a factores distintos del consumo de cannabis, como la propia adolescencia, que conlleva manifestaciones de indolencia (se ha hablado de letargo puberal), o bien los rasgos de personalidad ya existentes, los diversos factores socioculturales que puedan favorecer la pasividad, así como el consumo concomitante de otros psicofármacos. A su vez, podría plantearse la hipótesis de que los consumidores amotivados presentan una mayor tendencia a recurrir al uso de la marihuana precisamente porque se sienten apáticos y fracasados, hecho que explicaría que diferentes estudios no hayan encontrado diferencias significativas entre los adolescentes universitarios que consumen cannabis y los que no lo hacen.

Al margen de la posible etiología de este síndrome amotivacional, si es que existe, la realidad clínica nos presenta a un grupo de personas con gran dificultad para desarrollar una vida diaria lo suficientemente gratificante como para no reiniciar el consumo, sobre todo cuanto menor es su edad de inicio. El cannabis normalmente dificulta el proceso de maduración del individuo, sobre todo cuando el consumo ha comenzado en la adolescencia, que es la etapa vital en la que el individuo aprende a adoptar una conducta activa ante los problemas. Desde esta perspectiva se explica el hecho de que los consumidores habituales puedan presentar dificultades para plantearse objetivos, quizá por menor autoestima que la de los no consumidores.

Para concluir, cuando la asistencia del paciente se vuelve intermitente y con escasa motivación para proseguir con el tratamiento, se aconseja una actitud de aceptación del hecho. Dicha aceptación implica mostrarse disponible para recuperar la relación si el usuario decide su reinicio. Aunque la inmensa mayoría de los pacientes deshabituados vuelven a fumar cannabis -en nuestra experiencia prácticamente todos-, se espera de ellos que no lo hagan con tanta intensidad, es decir, que pasen a ser consumidores sobrios.

\subsection{Abordaje grupal}

El consumo de marihuana o de hachís, como el de tabaco, suele iniciarse dentro del propio grupo de pares, en edades tempranas y con un escaso conocimiento de las posibles consecuencias negativas que puede entrañar a medio o largo plazo. Cabría hablar, por tanto, de un típico ejemplo de presión grupal, que suele acentuarse cuando el grupo es un referente más importante para el joven que la propia familia (cuadro 10).

Las sesiones terapéuticas grupales ofrecen un marco muy adecuado para el entrenamiento en técnicas de relajación así como para la prevención de otras posibles drogodependencias y la prevención de recaídas, puesto que rentabilizan las intervenciones y favorecen la adquisición de habilidades sociales que después se extrapolarán a otro tipo de situaciones (Liddle, 1998).

Las intervenciones grupales deberían partir, en teoría, de una adecuada selección de 


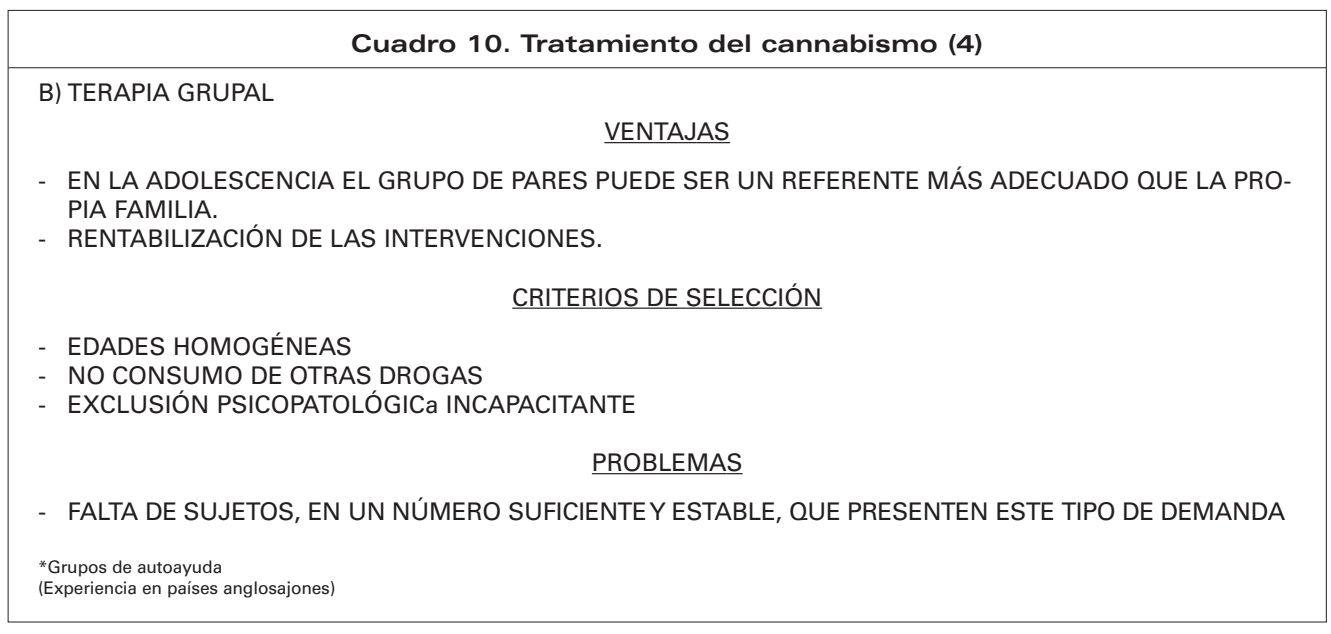

sus participantes, adoptando los criterios más adecuados en función de los objetivos perseguidos. He aquí tres criterios de selección exigentes:

1) Exclusión de psicopatologías incapacitantes. En caso de diagnósticos duales (abuso de sustancias junto con trastornos psíquicos) se prioriza el seguimiento individualizado. El equipo terapéutico siempre hará una valoración personal de cada caso.

2) Edades homogéneas: sujetos con un alto historial de consumo no deberían coincidir con adolescentes recién iniciados, puesto que las problemáticas asociadas tienen pocos puntos en común y por posible riesgo de resultados contraterapéuticos.

3) Ausencia de consumos asociados de otras drogas ilegales, pero no de tabaco y alcohol.

En la práctica, como sucede en los grupos terapéuticos con pacientes alcohólicos o tabaquistas, hay autoselección, tanto espontánea del paciente como orientada por el terapeuta que lleva el grupo.

Al margen de los grupos terapéuticos de carácter estructurado y que suelen estar dirigidos por un profesional, también existen grupos de autoayuda para consumidores de cannabis en los países anglosajones. En nuestro entorno no tenemos noticia de ninguno. Los grupos de autoayuda se han des- arrollado a partir de las experiencias de los ya clásicos grupos de Alcohólicos Anónimos. Se basan en el principio del apoyo mutuo entre personas con un mismo problema, e inciden mucho en los aspectos emocionales de la relación persona-sustancia.

\subsection{Abordaje familiar}

Cuando un profesional de la salud sospecha que un menor de edad está utilizando marihuana o hachís, debería confirmar el diagnóstico y valorar los resultados tanto con el posible consumidor como con sus padres. Debería utilizar un discurso firme, de apoyo, y por supuesto de índole no judicial. La intervención debería aportar referentes apropiados para la rehabilitación, así como propiciar un seguimiento posterior del caso (cuadro 11).

La educación global a la familia acerca de lo que representa el consumo de tóxicos y sus riesgos, y la monitorización clínica del paciente en aras de la consecución de la abstinencia cannábica son los primeros pasos para poder concluir con éxito el proceso de rehabilitación. Gran parte de las reglas del sistema familiar especifican la manera en que el joven y allegados deben actuar, puesto que la familia modela, canaliza y delimita la conducta del joven (CSAT, 1998). 


\begin{tabular}{|l|}
\multicolumn{1}{|c|}{ Cuadro 11. tratamiento del cannabismo (5) } \\
\hline C) INTERVENCIÓN FAMILIAR \\
1. ASESORAMIENTO-INFORMACIÓN \\
- ELEVADO NÚMERO DE DEMANDAS EN LOS SERVICIOS DE INFORMACIÓN \\
- OBJETIVOS DE LA INTERVENCIÓN: \\
- antextualización y desdramatización del hecho \\
- evitar actitudes comportamentales \\
- evitar exceso de informactectión \\
- si elevado nivel de ansiedad; $2^{\text {a }}$ intervención \\
2. Orientación del caso \\
FamERVENCIÓN FAMILIAR \\
PAUTA. red de apoyo social básica \\
1. Normas coherentCAS DE INTERACCIÓN FAMILIAR: \\
2. Entrenamiento habilidades comunistos miembros \\
3. Contrato contingencias entre las partes afectadas \\
Posible utilización de las analíticas de orina como: \\
• forma objetiva de control \\
• medio restablecer la confianza
\end{tabular}

Dentro del amplio espectro de intervenciones con o para la familia que se desarrollan en un servicio para drogodependientes, en los casos de fuerte consumo de cannabis hay, como mínimo, dos niveles diferentes de actuación: por un lado, el asesoramiento a la familia, y por otro, mucho más complejo, la integración de la familia dentro del tratamiento del consumidor. Veamos cada uno de ellos:

\subsection{Intervención informativa:}

No son pocas las demandas generadas por padres $u$ otros familiares en relación a la posible sospecha o falta de información sobre un posible consumo de porros por parte del hijo/a, normalmente adolescente. La consulta suele iniciarse con los siguientes términos: "encontré una barrita envuelta en papel de plata en el bolsillo del pantalón," “|legó a casa con los ojos enrojecidos", "va con amigos que fuman porros", etc.

En esta situación, el primer objetivo es el de lograr, en la medida de lo posible, una desdramatización del hecho, valorando si se han producido o no cambios relevantes en la conducta del paciente potencial. Areas importantes a analizar son: nuevas amistades, rendimiento escolar y/o laboral, actitudes con la familia, incrementos en el gasto de dinero, cambios de la imagen externa y del carácter, cambio en los horarios, etc.

En caso afirmativo en uno o varios de estos ítems, las sospechas iniciales deberán indagarse con mayor profundidad. Sin embargo, hay que insistir en el hecho de que los padres deben actuar como tales y no como pseudodetectives, partiendo de que una posición excesivamente represiva o inquisidora puede producir el efecto contrario al deseado. En muchas ocasiones es conveniente reconducir a los padres a otras posibles vías de exploración, dirigiéndolos a los profesores o a los amigos del hijo, los cuales tienden a presentar una visión más objetiva de la situación por no llevar la carga emocional propia de los progenitores.

En el primer contacto, si la familia lo solicita, habrán de abordarse las posibles consecuencias, a corto y medio plazo, del consumo de cannabinoides, ofreciendo una alternativa de tratamiento para el afectado, en caso de que sea necesario. En este punto hay que valorar los pros y los contras de la intervención o no en un servicio de drogodependencias. Por supuesto, hay que descartar previamente que el consumo sólo haya sido un hecho esporádico en la vida del sujeto, sin otras consecuencias asociadas, ya que entonces es ridículo intervenir. 
Lo que nunca debe hacerse es sobrecargar a los allegados con una cantidad mayor de información de la que éstos puedan asimilar. Queda claro que en circunstancias como éstas no es bueno ofrecer clases magistrales. En un primer contacto siempre será preferible programar una segunda intervención a dejarse llevar por la ansiedad de los padres, que tienden a querer arreglarlo todo milagrosamente de una sola vez. El profesional de la terapia no debe correr el riesgo de hablar en exceso y proferir juicios precipitados.

\subsubsection{Intervención terapéutica:}

La familia juega un papel importante en el desarrollo de la motivación del paciente: constituye su red de apoyo social básica. Por ello, será preciso establecer de forma conjunta pautas específicas de interacción familiar que favorezcan la buena marcha del proceso de recuperación. Entre ellas destacan:

1) normas de actuación familiar, que deben ser coherentes entre los distintos miembros de la familia;

2) entrenamiento en habilidades de comunicación, que suelen distorsionarse durante el tiempo de dependencia; y

3) contrato de contingencias o acuerdos entre las partes afectadas, para lo cual pueden utilizarse las analíticas de orina como forma objetiva de control y de ir restableciendo la confianza de la familia en el paciente (NIDA, 1998).

\section{CONTROLES DE ORINA}

En el organismo, la lenta farmacocinética de los cannabinoides nos lleva a una importante conclusión: que los controles de orina o urinálisis para detectar estos metabolitos ofrecen una significación muy relativa. Si antes veíamos que podemos adaptarnos a ella con fines terapéuticos, con fines fiscalizadores los controles de orina para detección de cannabinoides apenas sirven.
En efecto, que un sujeto dé positivo a cannabinoides nada nos dice sobre si el consumo tuvo lugar recientemente o hace varios días e incluso semanas. Por lo tanto, es bastante discutible plantear la necesidad de una estrategia de control en términos preventivos o de disuasión. Esto ha sido propuesto en el puesto de trabajo, en tareas de riesgo como pilotar aviones, conducción de trenes y autobuses, etc. Dicha necesidad quedaría doblemente refutada. En primer lugar porque un positivo de cannabis puede corresponder a un uso pretérito, tal como se ha visto. En segundo lugar, porque un resultado negativo de cannabinoides en orina no es ninguna garantía de que no se produzca un flashback o episodio de reviviscencia en pleno trabajo, con el eventual riesgo, por muy improbable que esto sea. Por tanto, monitorizar cannabinoides con repetidos urinálisis constituye una indicación racional solamente si los fines son terapéuticos.

En la práctica, pues, nunca debemos olvidar que un único consumo puede causar cannabinolemia persistente incluso tras más de dos semanas de dicha autoadministración aguda, y que el usuario crónico puede dar positivo pasado un mes o incluso más después de haber interrumpido el consumo. Hay que poner en duda, pues, la utilidad de ciertos protocolos de control como los preconizados por algunas aerolíneas con sus trabajadores: test al alta laboral, en la visita anual, tras accidentes laborales, tras ausencias no breves -más de 21 días, por ejemplo-, y después de ausencias repetidas.

Al margen del debatido status jurídico de la imposición de un control, es evidente que los urinálisis no se adaptan bien al cannabis, pues la detección no se circunscribe al consumo inmediato. La detección queda innecesariamente ampliada a un consumo remoto que no hay porqué juzgar en relación a lo que motiva el control (a menos que haya habido un compromiso previo en dicho sentido). En cambio, para otras drogas como alcohol, benzodiacepinas, heroína, o cocaína, hay mayor adecuación farmacocinética entre estos controles y las sustancias a detectar. 
Por último, es evidente que en los centros de nuestro país especializados en toxicomanías, tanto ambulatorios como hospitalarios, la detección sistemática de cannabinoides y otras drogas de abuso ha de ser rutinaria. En un marco clínico general, este screening o cribado sólo tendría sentido, como máximo, en drogodependientes, demás enfermos psiquiátricos, y pacientes de difícil diagnóstico, en especial entre adolescentes y adultos jóvenes.

A efectos prácticos, la monitorización del paciente cannábico suele realizarse con tecnología aceptablemente sensible, pero no todo lo específica que quisiéramos, pues habitualmente no se distingue entre cannabinoides de distinta farmacocinética.

\section{CONCLUSIONES}

El tratamiento del paciente consumidor de cannabis no se circunscribe al tratamiento de las secuelas psiquiátricas (ansiedad, psicosis) que ya se viene haciendo, sino que debería incluir el tratamiento de cesación del hábito. Las técnicas de cesación del hábito tabáquico han de ser convenientemente adaptadas y son de elección en quienes demandan esta ayuda concreta; hallan cabida en este trabajo. Se justifica porque: nicotina y cannabinoides son sustancias de abuso masivamente fumadas, su respectivo status social va aproximándose, están sujetas a la misma ambivalencia atractiva-aversiva de todo fármaco/droga y al correspondiente antagonismo social prohibición-tolerancia, y sobre todo porque la dependencia que potencialmente generan requiere una oferta asistencial completa y continuada como proceso terapéutico que incluya el tratamiento psicosocial del hábito. Los controles de orina tienen indicación terapéutica en dicho proceso, pero no fiscalizadora por la lenta cinética de los cannabinoides.

\section{BIBLIOGRAFÍA}

CIE-10 (1992) Clasificación Internacional de las Enfermedades, Organización Mundial de la Salud, $10^{\mathrm{a}}$ revisión, Meditor, Madrid.

CSAT (1998) Center for Substance Abuse Treatment: Adolescent substance abuse. Assessment and treatment, Treatment Improvement Protocol Series (TIPS), National Institute on Drug Abuse, Rockville (Md).

DSM-IV (1995) Diagnostic and Statistical Manual of Mental Disorders, forth edition, American Psychiatric Association, 1994. Manual diagnóstico y estadístico de los trastornos mentales, $4^{\text {a }}$ edición, Masson, Barcelona.

Becoña, E (1998) Tratamiento del tabaquismo, en Caballo VE (dir.) Manual para el tratamiento cognitivo-conductual de los trastornos psicológicos vol. 2, Siglo XXI, Madrid.

Liddle HA (1998) Multidimensional family therapy treatment manual, Center for Treatment Research on Adolescent Drug Abuse, University of Miami School of Medicine

NIDA (1998) National Institute on Drug Abuse: Therapy Manual for Drug Addiction, Rockville (Md).

Smith DE (1968) Acute and chronic toxicity in marijuana, Journal for Psychedelic Drugs 2.

Solé Puig, J (2000 a) Drogas, leyes y Platón (I), Jano 4-10 febrero vol 53, núm 1330

Solé Puig, J (2000 b) Drogas, leyes y Platón (II), Jano 11-17 febrero vol 53, núm 1331

Solowij N (1998): Cannabis and cognitive functioning, Cambridge University Press, Cambridge (Ma).

Tennant FS (1986) The clinical syndrome of marijuana dependence, Psychiatric Annals 16, 225232.

USDHHS, U.S. Department of Health and Human Services (1997) Marijuana, información para los adolescentes. NIH publication number 95-4037, Rockville (Md).

USDHHS, U.S. Department of Health and Human Services (1998) La marijuana: lo que los padres deben saber. 